\title{
Short communication: The role of autoinducer 2 (Al-2) on antibiotic resistance regulation in an Escherichia coli strain isolated from a dairy cow with mastitis
}

\author{
Ting Xue, ${ }^{1}$ Lumin Yu, Fei Shang, Wenchang Li, Ming Zhang, Jingtian Ni, and Xiaolin Chen ${ }^{1}$ \\ School of Life Sciences, Anhui Agricultural University, Hefei, Anhui 230036, China
}

\begin{abstract}
Extended spectrum $\beta$-lactamase (ESBL)-positive Escherichia coli is a major etiological organism responsible for bovine mastitis. The autoinducer 2 (AI-2) quorum sensing system is widely present in many species of gram-negative and gram-positive bacteria and has been proposed to be involved in interspecies communication. In $E$. coli model strains, the functional mechanisms of AI-2 have been well studied; however, in clinical antibiotic-resistant E. coli strains, whether AI-2 affects the expression of antibiotic resistance genes has not been reported. In this study, we report that exogenous AI-2 increased the antibiotic resistance of a clinical $E$. coli strain isolated from a dairy cow with mastitis by upregulating the expression of TEM-type enzyme in an LsrR (LuxS regulated repressor)-dependent manner. Key words: Escherichia coli, mastitis, autoinducer 2 (AI-2) quorum sensing, extended spectrum $\beta$-lactamase
\end{abstract}

\section{Short Communication}

Quorum sensing (QS) is a process in which bacteria use chemical molecules as a signaling language to change behaviors to adapt to specific environments (Fuqua et al., 1994; Bassler, 1999, 2002; Miller and Bassler, 2001). It has been reported that QS bacteria with high density in culture exhibit decreased susceptibility to antibiotics, reducing the responsiveness to antibiotics (Liu et al., 2000; Spoering and Lewis, 2001; Roberts and Stewart, 2005). Autoinducer 2 (AI-2), which is produced by many species of gram-negative and gram-positive bacteria, is thought to be a QS signaling molecule involved in interspecies communication (Federle and Bassler, 2003; Sun et al., 2004; Vendeville et al., 2005; De Keersmaecker et al., 2006). Autoinducer 2 signals are a set

Received October 19, 2015.

Accepted March 2, 2016.

${ }^{1}$ Corresponding authors: xuet@ahau.edu.cn and cxlzyx@ahau.edu. $\mathrm{cn}$ of related molecules that are derived from spontaneous rearrangement of dihydroxy-2,3-pentanedione (DPD; Winzer et al., 2002; Miller et al., 2004). It has been reported that AI-2 QS affects antibiotic susceptibility in Streptococcus anginosus and Staphylococcus aureus (Ahmed et al., 2007; Xue et al., 2013). In Escherichia coli model strains, the functional mechanisms of AI-2 have been well studied (Wang et al., 2005; Xue et al., 2009); however, these model strains generally do not contain antibiotic resistance genes. Whether AI-2 affects the expression of antibiotic resistance genes in clinical antibiotic-resistant $E$. coli strains has not been reported.

The $\beta$-lactam groups of antibiotics such as penicillin or ampicillin have been the drugs of choice for treatment of bacterial infections. However, the emergence of the extended spectrum $\beta$-lactamase (ESBL) resistance mechanism brought a great challenge for the common use of $\beta$-lactam antibiotics and routine antimicrobial therapy (Jacoby and Medeiros, 1991; Bush, 2001; Altayb et al., 2014). In this study, strain ECDCM1 was a clinical $E$. coli strain isolated from a dairy cow that suffered from mastitis. The MIC assays showed that strain ECDCM1 was resistant to several $\beta$-lactam antibiotics, including ampicillin, oxacillin, and penicillin. The TEM-type ESBL are recognized as one of the most prevalent mechanisms of resistance to $\beta$-lactam antibiotics in the Enterobacteriaceae (Bonnet et al., 1999; Pai et al., 2001). The multi-antibiotic resistance of strain ECDCM1 is likely related to TEM. Thus, the genomic DNA of strain ECDCM1 was extracted and the TEM gene was detected by PCR. As expected, the TEM gene was amplified successfully and the fragments were sequenced. According to the sequencing results, strain ECDCM1 contains the typical TEM-type gene TEM-1.

Growth assays were performed according to the following methods. Colonies of strain ECDCM1 were placed into $2 \mathrm{~mL}$ of Mueller-Hinton (MH) broth (Oxoid, Basingstoke, UK) and cultivated at $37^{\circ} \mathrm{C}$ with shaking at $200 \mathrm{rpm}$ for $16 \mathrm{~h}$. Then, the overnight cultures were inoculated into $20 \mathrm{~mL}$ of fresh $\mathrm{MH}$ medium and diluted 
Table 1. Oligonucleotide primers $(\mathrm{F}=$ forward; $\mathrm{R}=$ reverse $)$ used in this study

\begin{tabular}{ll}
\hline Primer name & Oligonucleotide $\left(5^{\prime}-3^{\prime}\right)$ \\
\hline rt-16s-f & F: TTTGAGTTCCCGGCC \\
rt-16s-r & R: CGGCCGCAAGGTTAA \\
rt-TEM-f & F: AGAGTTTTCGCCCCG \\
rt-TEM-r & R: CCCCCATGTTGTGCA \\
rt-lsrR-f & F: CGGATCGCGTGGTTT \\
rt-lsrR-r & R: TCAACATATGCGCCGC \\
g-TEM-f & F: ATGAGTATTCAACATTTTCG \\
g-TEM-r & R: TTACCAATGCTTAATCAGTG \\
\hline
\end{tabular}
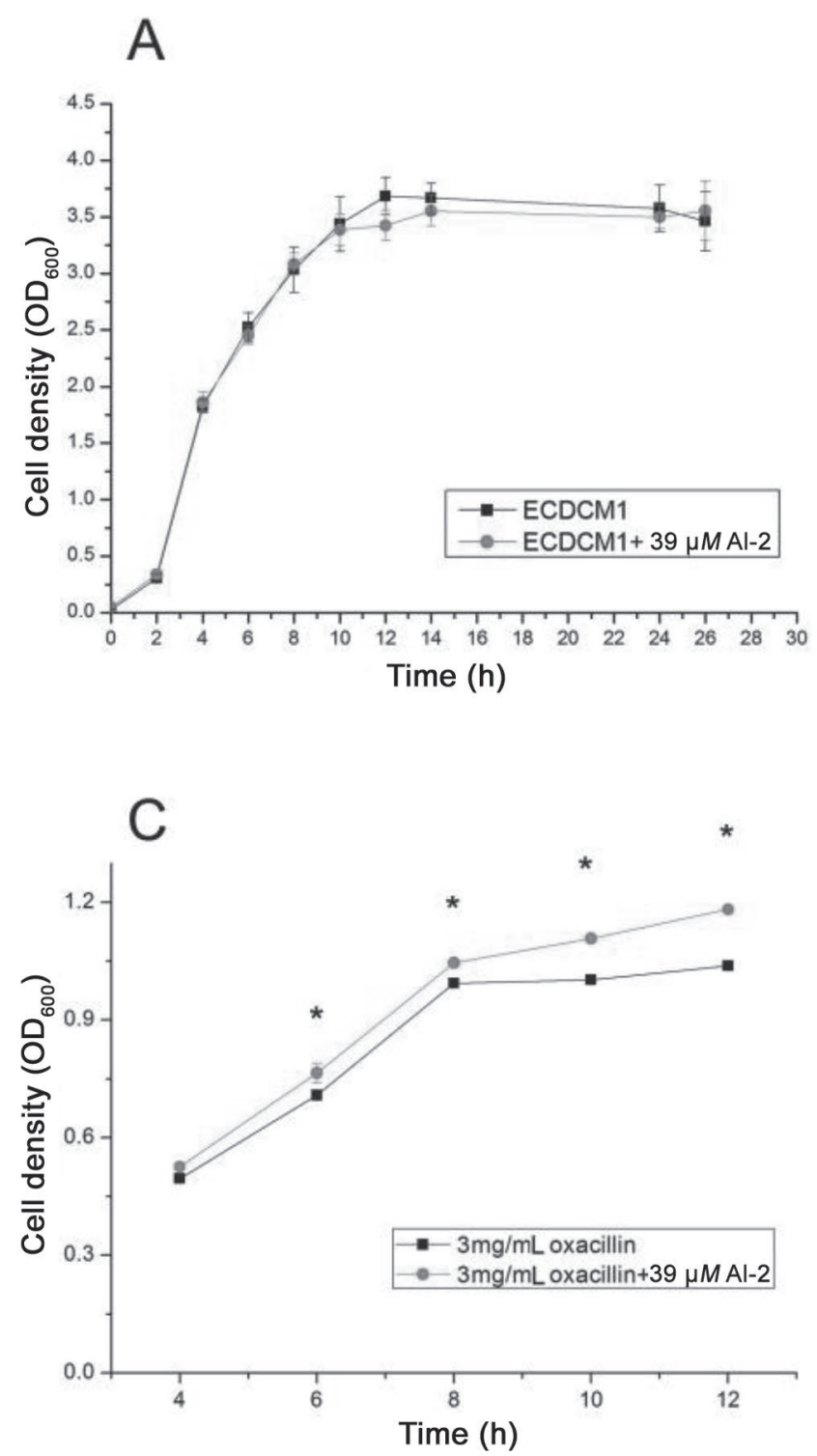

to a final concentration of $\mathrm{OD}_{600}$ (optical density at 600 nm) 0.05 and, when necessary, ampicillin, oxacillin, or penicillin was added. The cell density was detected at each time point by using a UV/Vis spectrophotometer (DU730; Beckman Coulter, Miami, FL).

Antibacterial assays were performed according to a previous study (Chen et al., 2015). The cells of strain ECDCM1 were first exposed to antibiotics with or without exogenous AI-2 for $12 \mathrm{~h}$ at $37^{\circ} \mathrm{C}$ and were then inoculated into fresh $\mathrm{MH}$ broth and spread onto $\mathrm{MH}$ agar plates. After culturing for $24 \mathrm{~h}$ at $37^{\circ} \mathrm{C}$, colony-
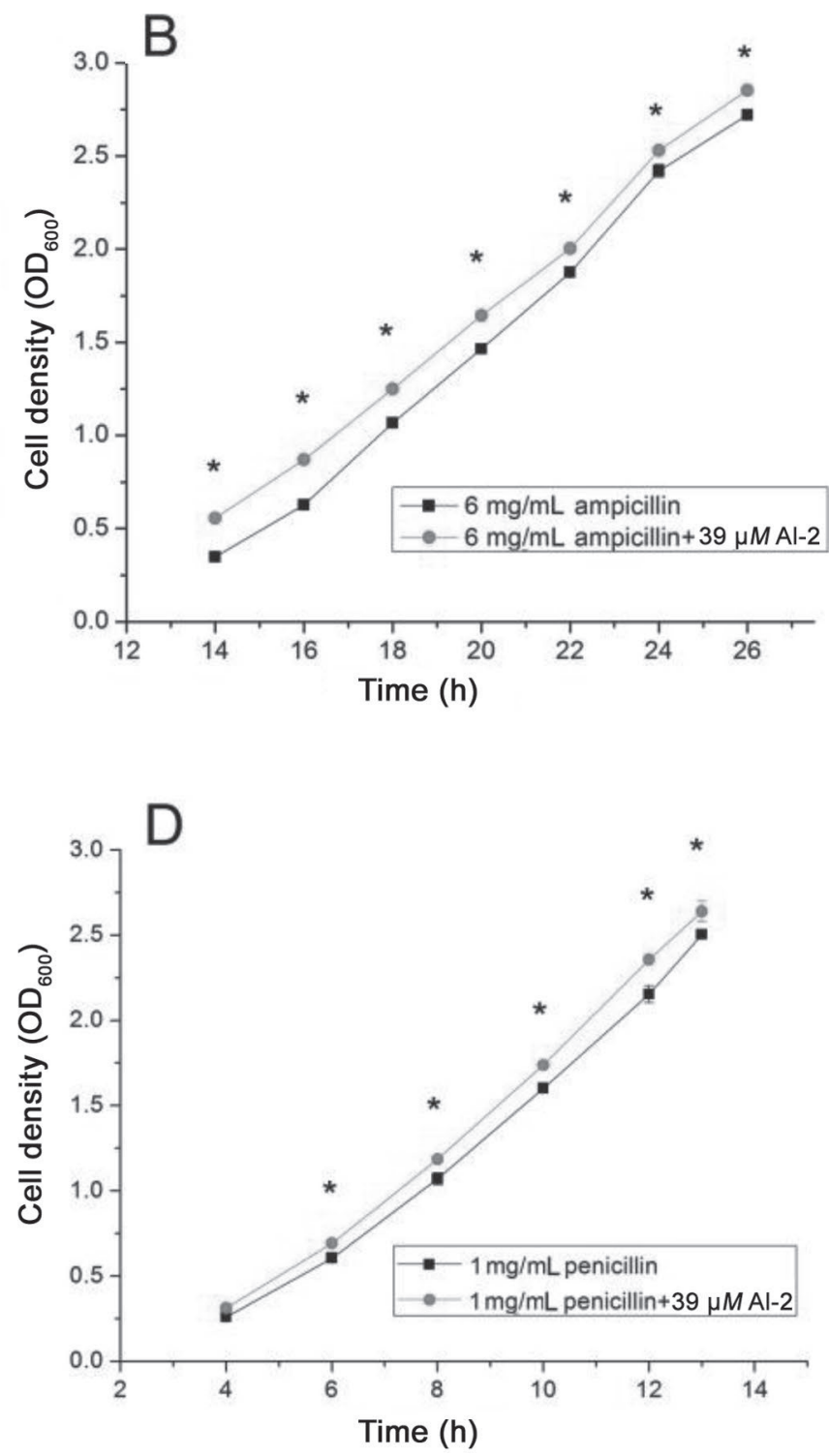

Figure 1. The growth curves (measured as optical density at $600 \mathrm{~nm}, \mathrm{OD}_{600}$ ) of Escherichia coli strain ECDCM1 and strain ECDCM1 cultured with $39 \mu M$ autoinducer 2 (AI-2) under different antibiotic conditions: (A) without any antibiotics, (B) with $6 \mathrm{mg} / \mathrm{mL}$ ampicillin, (C) with $3 \mathrm{mg} / \mathrm{mL}$ oxacillin, and (D) with $1 \mathrm{mg} / \mathrm{mL}$ penicillin G. Error bars indicate standard deviations. The results represent a mean of 3 independent experiments; ${ }^{*} P<0.05$, indicating a difference between antibiotic alone and antibiotic + AI- 2 . 
forming units were counted and viable colonies between the control and test group were compared. The microbial counts of the control group exposed to antibiotics only (no AI-2) were designated as $100 \%$.

The transcript levels of genes associated with antibiotic resistance and AI-2 were tested by performing real-time reverse transcription (RT)-PCR experiments. Cells were collected and resuspended in Tris-EDTA buffer ( $\mathrm{pH}$ 8.0) containing $10 \mathrm{~g} / \mathrm{L}$ lysozyme. After incubation for $5 \mathrm{~min}$ at $37^{\circ} \mathrm{C}$, total RNA was extracted from cells using the Trizol reagent (Ambion, Austin, TX), and residual DNA was removed using DNaseI (TaKaRa, Dalian, China). Real-time RT-PCR was performed using the PrimeScript 1st Strand cDNA synthesis kit, SYBR Premix Ex Taq (TaKaRa), and a StepOne realtime PCR system (Applied Biosystems, Carlsbad, CA). Difference of gene expression was calculated by $\Delta \Delta \mathrm{Ct}$ (where $\mathrm{Ct}=$ cycle threshold) method, using 16S gene as the housekeeping gene, normalized by subtracting the $\mathrm{Ct}$ value of $16 \mathrm{~S} \mathrm{cDNA}$ from target $\mathrm{cDNA}$. All primers used in this study are listed in Table 1 and had PCR efficiency between 1.93 and 2.09 .

The digoxigenin (DIG) gel shift assays were performed according to a previous study (Xue et al., 2009). The transcriptional regulator LsrR protein was overexpressed and purified according to the methods described previously (Xue et al., 2009) and the promoter region of TEM was amplified by PCR. The PCR products were labeled using the DIG gel shift kit (Roche, Indianapolis, IN) and were incubated at $25^{\circ} \mathrm{C}$ for $15 \mathrm{~min}$ with various amounts of LsrR protein. After incubation, the mixtures were electrophoresed in a $4.5 \%$ native polyacrylamide gel in $0.5 \times$ Tris-borateEDTA buffer. The band shifts were detected by the DIG gel shift kit.

All data were analyzed using the $t$-test ANOVA, with a $P$-value $<0.05$ considered significant. The experiments were repeated at least 3 times with similar results, and 1 representative result is shown.

To determine the effect of AI-2 on survival of strain ECDCM1 in the presence of antibiotics (ampicillin, oxacillin, and penicillin), cells of strain ECDCM1 were cultured in $\mathrm{MH}$ broth, and growth curves were detected under different conditions. The growth of strain ECDCM1 was first observed with the addition of exogenous AI-2 only, without any kind of antibiotics. The growth curve of strain ECDCM1 was similar to that of a test group cultured with exogenous AI-2 at $39 \mu M$ (Figure 1A). Subsequently, we evaluated the effect of AI-2 in the presence of antibiotics. Ampicillin, oxacillin, and penicillin were added to the culture medium at concentrations of 6,3 , and $1 \mathrm{mg} / \mathrm{mL}$, respectively. As shown in Figure 1B and Figure 1D, in the presence of ampicillin or penicillin, addition of exogenous AI-2
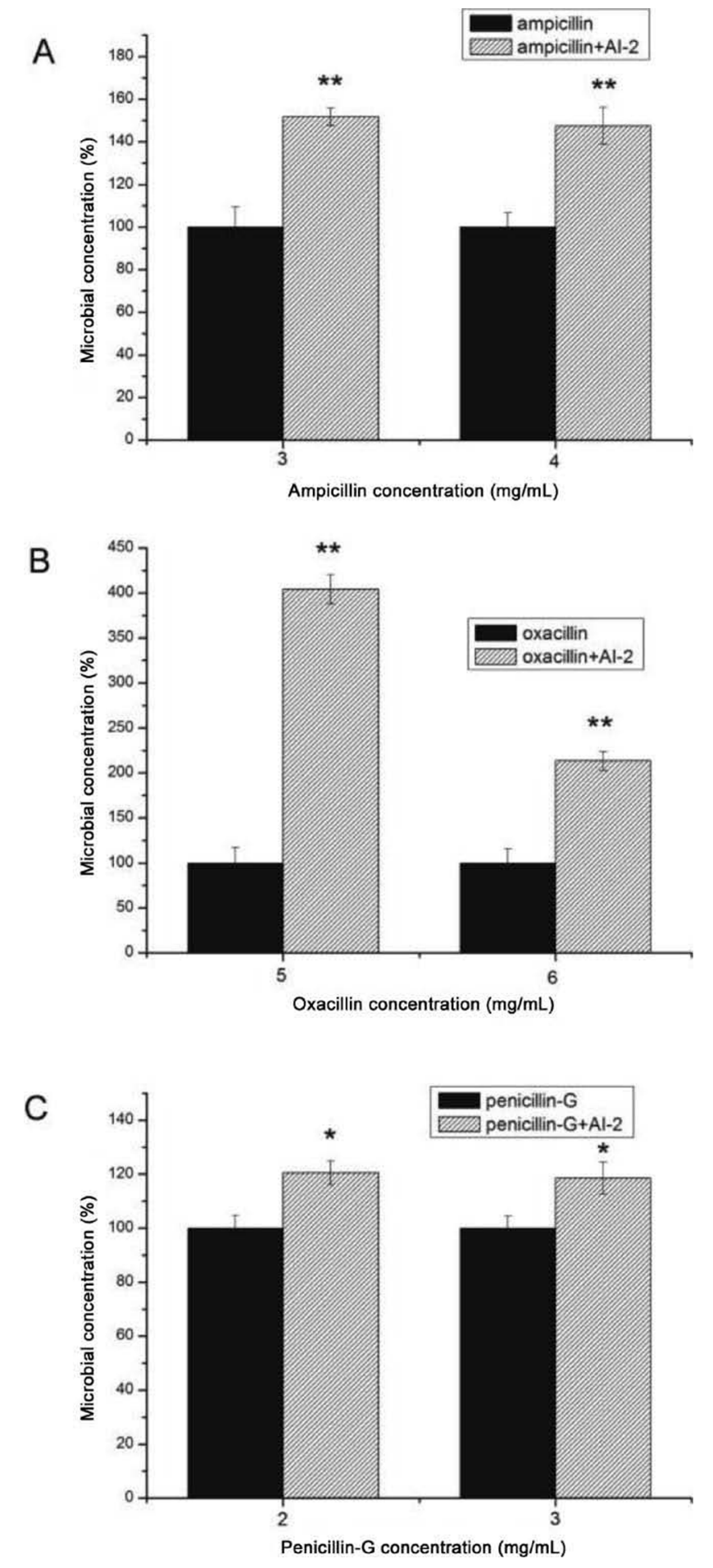

Figure 2. Colony-forming units assays of Escherichia coli strain ECDCM1 and strain ECDCM1 cultured with $39 \mu M$ autoinducer 2 (AI-2) under different antibiotic conditions. The survival rate of the control group without addition of AI-2 was designated 100\%. The colony counts of the test group cultured with AI-2 under different antibiotics $(\mathrm{A}=$ ampicillin, $\mathrm{B}=$ oxacillin, $\mathrm{C}=$ penicillin $\mathrm{G})$ were compared with that of the control group (without AI-2). Error bars indicate standard deviations. The results represent a mean of 3 independent experiments; ${ }^{* *} P<0.01,{ }^{*} P<0.05$, indicating a difference between antibiotic alone and antibiotic + AI-2. 
A

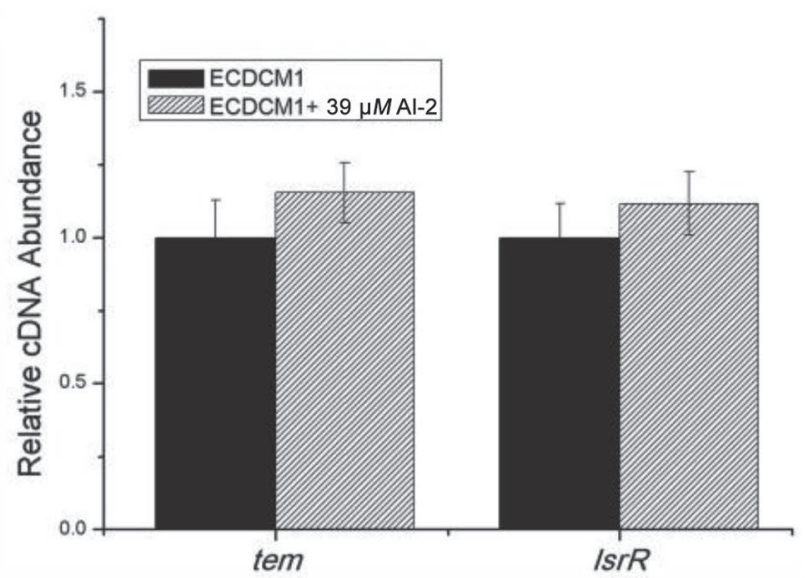

Antibiotic-free

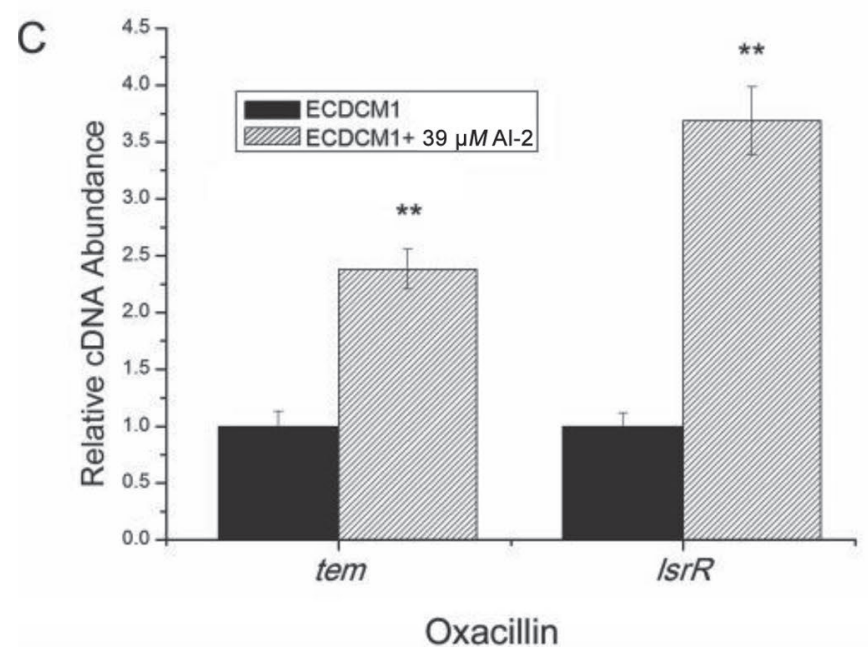

B

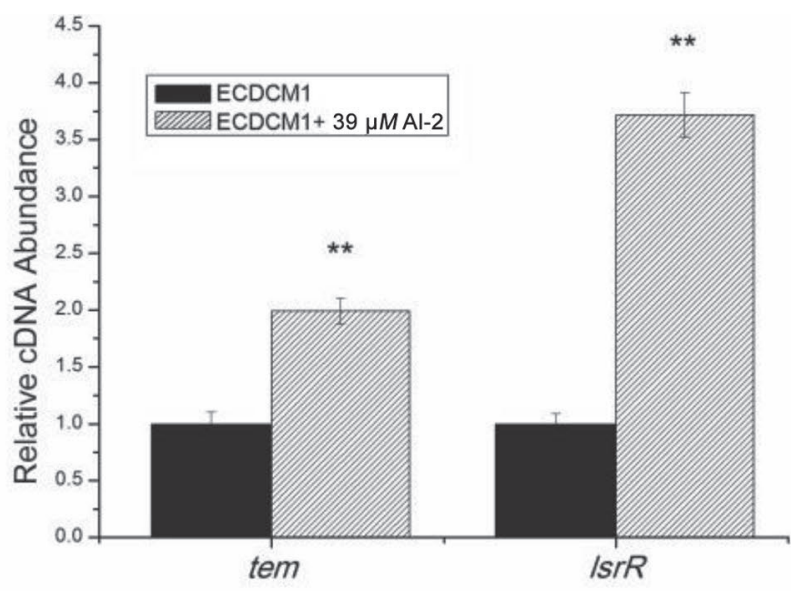

Ampicillin

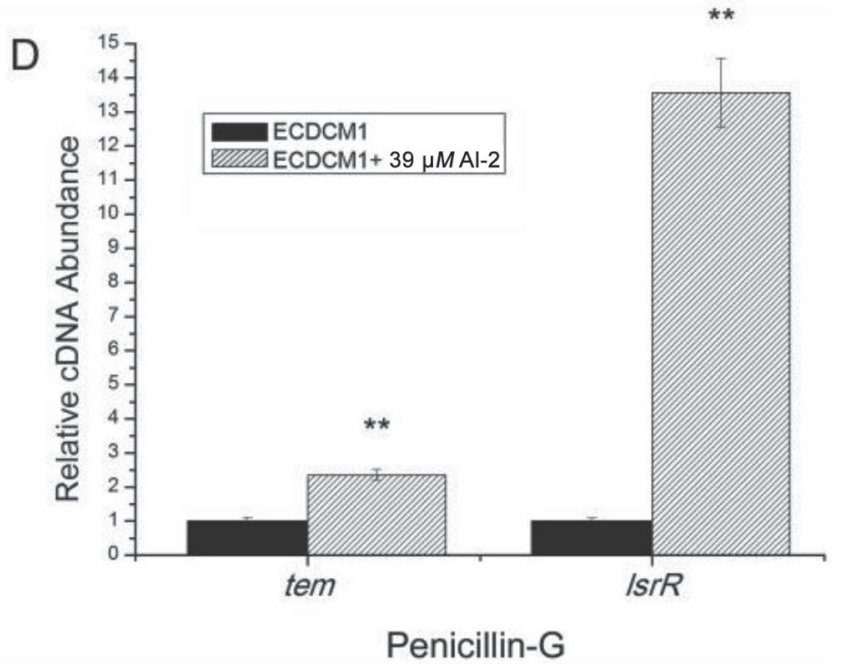

Figure 3. Comparative measurement of transcription (cDNA abundance) of TEM and lsrR in Escherichia coli strain ECDCM1. Relative transcript levels of TEM and $l s r R$ were tested by real-time reverse transcription-PCR in strain ECDCM1 and strain ECDCM1 cultured with 39 $\mu M$ autoinducer 2 (AI-2) under different antibiotic conditions: (A) without any antibiotics, (B) with $6 \mathrm{mg} / \mathrm{mL}$ ampicillin, (C) with $3 \mathrm{mg} / \mathrm{mL}$ oxacillin, (D) with $1 \mathrm{mg} / \mathrm{mL}$ penicillin G. Error bars indicate standard deviations. The results represent a mean of 3 independent experiments; ${ }^{* *} P<0.01$, indicating a difference between antibiotic alone and antibiotic + AI- 2 .

led to an increase of cell density of strain ECDCM1 at each culture time point. Moreover, in the presence of oxacillin, addition of AI-2 not only increased the cell density of the bacteria, but also increased the growth rate of the cells at $\mathrm{h} 10$ and 12 (Figure 1C). These results indicated that the antibiotic resistance of this strain increased upon addition of AI-2.

To further examine changes in the antibiotic resistance of strain with addition of exogenous AI-2, antibacterial assays were performed. The survival rates of the test groups with exposure to antibiotics and AI-2 were higher than that of the control group (Figure 2). The survival rate of the test group with oxacillin $(5$ $\mathrm{mg} / \mathrm{mL}$ ) and AI-2 was almost 4 times higher than that of the control group (Figure 2B). These results indicate that with addition of exogenous AI-2, resistance of the strain ECDCM1 to all 3 antibiotics increased, suggesting that this clinical E. coli isolate might use AI-2 as a signal to upregulate its antibiotic resistance.

To investigate the mechanism of how AI-2 affects resistance to antibiotics in E. coli, the transcript levels of genes associated with antibiotic resistance and AI-2 were assessed by performing real-time RT-PCR experiments. The TEM gene is important in $\beta$-lactam antibiotic resistance and is related to resistance to ampicillin, oxacillin, and penicillin in E. coli (Jacoby and Medeiros, 1991; Pai et al., 2001). The transcript levels of TEM and $l s r R$ were measured with or without ad- 


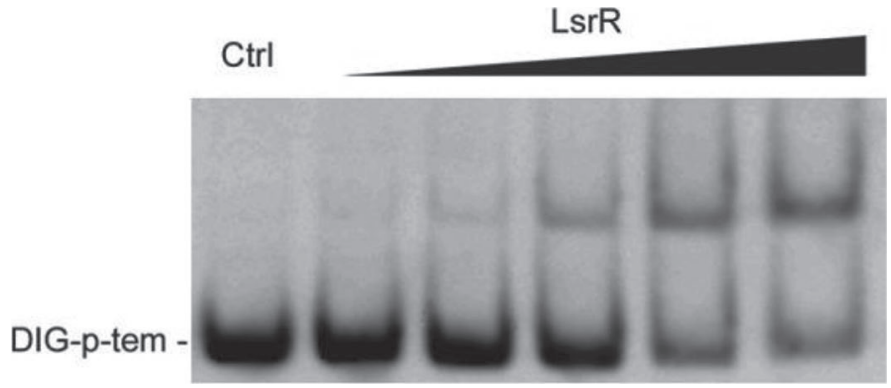

Figure 4. The binding ability of LsrR to the TEM promoter was determined by gel-shift assays. Increasing amounts of LsrR were incubated with digoxigenin (DIG)-labeled TEM promoters (DIG-p-TEM). In each panel, from lanes 1 to 6 , the amounts of LsrR were $4,0,0.5,1$, 2 , and 4 pmol, respectively; 50 fmol of DIG-labeled probe was added to all lanes. In lane 1, except for the labeled probes, 1 pmol of unlabeled probe was added as the competitive control (Ctrl).

dition of exogenous AI-2 and with different antibiotics. As shown in Figure 3A, the transcription of TEM was not apparently affected by the addition of exogenous AI-2 without the presence of antibiotics. However, as shown in Figure 3B, C, and D, in the presence of antibiotics, addition of AI-2 resulted in 2.1-fold (ampicillin), 2.4-fold (oxacillin), and 2.4-fold (penicillin) increases in transcription of TEM, suggesting that exogenous AI-2 might upregulate the antibiotic resistance of strain ECDCM1 through TEM. Moreover, the transcript level of $l s r R$, which encodes the receptor of AI-2, was measured. Our results showed that in the presence of antibiotics, the exogenous AI-2 led to 3.8-fold (ampicillin), 3.7-fold (oxacillin), and 13.6-fold (penicillin) increases in $l s r R$ transcription in strain ECDCM1, further supporting that, in ESBL-positive E. coli, AI-2 might regulate the antibiotic resistance gene TEM in an LsrR-dependent manner.

Because LsrR contains a helix-turn-helix (HTH) DNA binding domain and has been reported as a QS regulator that can regulate the transcription of a variety of genes by binding to their promoters, we hypothesize that LsrR regulates TEM transcription by binding directly to its promoter region. To determine whether LsrR can bind to the promoter of TEM, we performed DIG-gel shift assays. As shown in Figure 4, LsrR exhibited strong binding ability to the promoter of TEM, confirming that LsrR regulates TEM transcription by binding directly to its promoter region.

In conclusion, AI-2 can regulate the expression of TEM through the AI-2 receptor LsrR. Moreover, in the presence of antibiotics, the antibiotic-resistant $E$. coli strain exhibited stronger antibiotic resistance in response to an increase in cell density. These data indicate that long-term exposure to antibiotics may force this strain to evolve a better survival strategy.
The AI-2 QS system may be a very important system for adaptation to a changing environment in a range of gram-negative and gram-positive bacteria. Although the TEM-type ESBL are among the most common mechanisms for antibiotic resistance in $E$. coli, which genes can regulate TEM has seldom been reported. This study provides new clues to the TEM-regulating mechanism and suggests that a treatment other than antimicrobial treatment should be considered in ESBLpositive E. coli.

\section{ACKNOWLEDGMENTS}

This work was supported by the Project of Anhui Province Key Discipline of Biology (2014TSTD003; Anhui, China) and the Talent Project of Anhui Agricultural University (YJ2013-6; Anhui, China).

\section{REFERENCES}

Ahmed, N. A., F. C. Petersen, and A. A. Scheie. 2007. AI-2 quorum sensing affects antibiotic susceptibility in Streptococcus anginosus. J. Antimicrob. Chemother. 60:49-53.

Altayb, H. N., N. M. E. Amin, M. M. Mukhtar, M. A. Salih, and M. A. M. Siddig. 2014. Molecular characterization and in silico analysis of a novel mutation in TEM-1 beta-lactamase gene among pathogenic E. coli infecting a Sudanese patient. Afr. J. Microbiol. Res. 2:217-223.

Bassler, B. L. 1999. How bacteria talk to each other: Regulation of gene expression by quorum sensing. Curr. Opin. Microbiol. 2:582-587.

Bassler, B. L. 2002. Small talk. Cell-to-cell communication in bacteria. Cell 109:421-424.

Bonnet, R., C. De Champs, D. Sirot, C. Chanal, R. Labia, and J. Sirot. 1999. Diversity of TEM mutants in Proteus mirabilis. Antimicrob. Agents Chemother. 43:2671-2677.

Bush, K. 2001. New beta-lactamases in gram-negative bacteria: Diversity and impact on the selection of antimicrobial therapy. Clin. Infect. Dis. 32:1085-1089.

Chen, X., F. Shang, Y. Meng, L. Li, Y. Cui, M. Zhang, K. Qi, and T. Xue. 2015. Ethanol extract of Sanguisorba officinalis L. inhibits biofilm formation of methicillin-resistant Staphylococcus aureus in an ica-dependent manner. J. Dairy Sci. 98:8486-8491.

De Keersmaecker, S. C., K. Sonck, and J. Vanderleyden. 2006. Let LuxS speak up in AI-2 signaling. Trends Microbiol. 14:114-119.

Federle, M. J., and B. L. Bassler. 2003. Interspecies communication in bacteria. J. Clin. Invest. 112:1291-1299.

Fuqua, W. C., S. C. Winans, and E. P. Greenberg. 1994. Quorum sensing in bacteria: the LuxR-LuxI family of cell density-responsive transcriptional regulators. J. Bacteriol. 176:269-275.

Jacoby, G. A., and A. A. Medeiros. 1991. More extended-spectrum beta-lactamases. Antimicrob. Agents Chemother. 35:1697-1704.

Liu, X., C. Ng, and T. Ferenci. 2000. Global adaptations resulting from high population densities in Escherichia coli cultures. J. Bacteriol. 182:4158-4164.

Miller, M. B., and B. L. Bassler. 2001. Quorum sensing in bacteria. Annu. Rev. Microbiol. 55:165-199.

Miller, S. T., K. B. Xavier, S. R. Campagna, M. E. Taga, M. F. Semmelhack, B. L. Bassler, and F. M. Hughson. 2004. Salmonella typhimurium recognizes a chemically distinct form of the bacterial quorum-sensing signal AI-2. Mol. Cell 15:677-687.

Pai, H., H. J. Lee, E. H. Choi, J. Kim, and G. A. Jacoby. 2001. Evolution of TEM-related extended-spectrum beta-lactamases in Korea. Antimicrob. Agents Chemother. 45:3651-3653. 
Roberts, M. E., and P. S. Stewart. 2005. Modelling protection from antimicrobial agents in biofilms through the formation of persister cells. Microbiology 151:75-80.

Spoering, A. L., and K. Lewis. 2001. Biofilms and planktonic cells of Pseudomonas aeruginosa have similar resistance to killing by antimicrobials. J. Bacteriol. 183:6746-6751.

Sun, J., R. Daniel, I. Wagner-Dobler, and A. P. Zeng. 2004. Is autoinducer-2 a universal signal for interspecies communication: A comparative genomic and phylogenetic analysis of the synthesis and signal transduction pathways. BMC Evol. Biol. 4:36.

Vendeville, A., K. Winzer, K. Heurlier, C. M. Tang, and K. R. Hardie. 2005. Making 'sense' of metabolism: autoinducer-2, LuxS and pathogenic bacteria. Nat. Rev. Microbiol. 3:383-396.

Wang, L., J. Li, J. C. March, J. J. Valdes, and W. E. Bentley. 2005. luxS-dependent gene regulation in Escherichia coli K-12 revealed by genomic expression profiling. J. Bacteriol. 187:8350-8360.
Winzer, K., K. R. Hardie, N. Burgess, N. Doherty, D. Kirke, M. T. Holden, R. Linforth, K. A. Cornell, A. J. Taylor, P. J. Hill, and P. Williams. 2002. LuxS: Its role in central metabolism and the in vitro synthesis of 4-hydroxy-5-methyl-3(2H)-furanone. Microbiology 148:909-922.

Xue, T., L. Zhao, and B. Sun. 2013. LuxS/AI-2 system is involved in antibiotic susceptibility and autolysis in Staphylococcus aureus NCTC 8325. Int. J. Antimicrob. Agents 41:85-89.

Xue, T., L. Zhao, H. Sun, X. Zhou, and B. Sun. 2009. LsrR-binding site recognition and regulatory characteristics in Escherichia coli AI-2 quorum sensing. Cell Res. 19:1258-1268. 